\title{
Executive functioning and quality of life in acromegaly
}

\section{Emilia Solomon ${ }^{1,2}$ \\ Dumitru Brănișteanu',3 \\ Andrei Dumbravă ${ }^{4}$ \\ Radu Gheorghe Solomon ${ }^{5}$ \\ Lorànt Kiss ${ }^{5}$ \\ Mihai Glod' \\ Cristina Preda ${ }^{1,3}$}

'Faculty of Medicine, Grigore T. Popa University of Medicine and Pharmacy, lași, Romania; ${ }^{2}$ Department of Endocrinology, CF University Hospital, lași, Romania; ${ }^{3}$ Department of Endocrinology, "Sf. Spiridon" Hospital, lași, Romania; ${ }^{4}$ Faculty of Psychology, Alexandru Ioan Cuza University, lași, Romania; ${ }^{5}$ SUD, Faculty of Medicine, University Lucian Blaga, Sibiu, Romania
Correspondence: Dumitru Brănișteanu "Grigore T. Popa" University of Medicine and Pharmacy, No 16, Universității Street, lași, 700I I5, Romania Email dumitru_branisteanu@yahoo.com

This article was published in the following Dove Medical Press journal: Psychology Research and Behavior Management

\begin{abstract}
Introduction: Active acromegaly is a rare chronic endocrine disorder caused by excessive growth hormone $(\mathrm{GH})$. Clinical studies suggest that cognitive performance is impaired in acromegaly - particularly executive function as well as short- and long-term memory. This study compared the quality of life (QoL) and executive functioning in acromegaly patients vs healthy controls.
\end{abstract}

Materials and methods: This was an observational case-control study on 38 subjects divided into 19 acromegaly patients and 19 matched controls. The groups were evaluated for QoL, attention, and executive function. All subjects completed Acromegaly Quality of Life Questionnaire (AcroQoL), Trail Making Test (parts A and B), Stroop, and phonemic fluency tests.

Results: Acromegaly patients had an AcroQoL global score that was significantly lower than controls. There were significant differences between the acromegaly group and the control group in terms of the physical effects $(P=0.001)$ and appearance $(P<0.001)$ but not for personal relationships $(P=0.421)$. Acromegaly patients performed worse in the trail making test part $\mathrm{B}$. They provided significantly fewer words than healthy subjects in phonemic fluency testing. Although patients performed generally worse than controls, no significant differences were noted in the trail making test part A, Stroop test, and the constrained phonemic fluency.

Conclusion: Acromegaly patients display worse executive functioning than healthy controls and have a decreased QoL.

Keywords: acromegaly, cognition, growth hormone, executive function

\section{Introduction}

Acromegaly is a rare chronic illness caused in $>95 \%$ of the cases by excessive secretion of growth hormone $(\mathrm{GH})$ due to pituitary adenoma; it is associated with increased mortality and morbidity. ${ }^{1-3}$ The impact of GH and IGF-1 on regulating brain functions and their neurotrophic role has been recently documented. ${ }^{4}$ IGF-1 has an important role in cognition. It improves cerebral circulation, increases neuronal activity, and inhibits neuronal apoptosis. ${ }^{5,6}$ The prefrontal cortex, hippocampus, and limbic structures have many GH and IGF-1 receptors. ${ }^{7,8}$ This may explain the decrease in executive function - the prefrontal cortex is responsible for this complex neuropsychological construct. "Dysexecutive syndrome" is usually associated with difficulties in setting goals, having future-oriented behavior, as well as planning, organizing, and flexible thinking during problem solving. ${ }^{9-12}$

In healthy condition, GH and IGF-1 have protective effects on the cognitive (eg, memory) and mood brain functioning. ${ }^{13}$ Moreover, clinical studies have demonstrated that both memory and general cognitive functions are improved when a patient with pituitary deficiency is successfully treated with recombinant GH. ${ }^{14-16}$ 
On the other hand, supraphysiological levels of GH and IGF-1 are correlated with cognitive dysfunction, but no pathological mechanistic associations have yet been discovered. One of the hypotheses is that excessive levels of brain IGF-1 might cause insulin resistance of the cerebrum leading to hyperphosphorylation and amyloid accumulation. These phenomena result in synaptic loss. ${ }^{17}$

There are many brain regions with a higher density of GH and IGF-1 receptors, including the hippocampus and the prefrontal cortex. These areas seem to be particularly vulnerable in acromegaly. ${ }^{7,8}$ Given the fact that these brain regions are essential for specific neurocognitive functioning, we hypothesize that patients diagnosed with acromegaly may suffer from impaired executive function. The purpose of the study was to evaluate the executive function and quality of life (QoL) in acromegaly patients compared to healthy controls. Our goal was to indicate that acromegaly is associated with impaired executive functioning and this decreases QoL in acromegaly sufferers.

\section{Materials and methods Study design}

This was a cross-sectional case-control study that assessed the executive functioning and the QoL of patients with acromegaly vs healthy subjects.

\section{Subjects}

Both the acromegaly and the control groups included 19 subjects, 14 women and five men. Volunteers were matched for age, sex, and education. The participants from the acromegaly group were recruited between January 2015 and February 2016 from the Endocrinology Department of Saint Spiridon Academic Hospital of Iasi. To eliminate confounding variables, we excluded those with current diagnosis or history of any of the following: 1) stroke; 2) Parkinson's disease; 3) traumatic brain injury; 4) major psychiatric disorders (including schizophrenia, dementia, and generalized anxiety disorders); 5) smoking, drug or alcohol abuse; 6) diabetes mellitus; or 7) uncontrolled systemic arterial hypertension.

The acromegaly group consisted of patients seeking inpatient endocrinology treatment. In total, 27 acromegaly patients were initially invited to participate in the study. Four refused, one patient was excluded due to a stroke, two did not complete all the tests, and one patient was illiterate.

The control group was represented by healthy volunteers - mostly relatives of the patients. The inclusion criteria were the absence of current systemic pathology such as systemic arterial hypertension, diabetes mellitus, or other metabolic or endocrine disturbances, as well as present or previous drug abuse.

All the patients gave informed written consent to participate in the study.

The diagnosis of active acromegaly was based on clinical features, biological parameters ( $\mathrm{GH}$ profile during oral glucose-tolerance test [OGTT] over 2 hours and IGF-1), and pituitary neuroimaging. Biochemically controlled acromegaly was based on the following two criteria: 1) $\mathrm{GH}$ levels $<1 \mu \mathrm{g} / \mathrm{L}$ during the OGTT and 2) IGF-1 levels within 2 SD of age and gender-adjusted norms. The global function of the anterior pituitary was also assessed (prolactin, adrenocorticotropic hormone [ACTH], thyroid-stimulating hormone [TSH], follicle-stimulating hormone [FSH], luteinizing hormone [LH] along with cortisol, thyroxine, and estradiol/testosterone).

\section{Assessment instruments}

QoL in acromegaly was assessed using the Acromegaly Quality of Life Questionnaire (AcroQoL). It contained 22 questions with five possible responses scored on a Likert-type scale (from 1 to 5) that evaluates physical and psychological domains. The psychological domain is subdivided into two subscales regarding the appearance and personal relationships. For each, a lower score is associated with a worse QoL.

We applied the following instruments to evaluate the executive functioning:

- Trail making test (TMT) part A estimates attention, motor speed, and visual research skills. Part B assesses higher cognitive functions such as mental flexibility, ability to execute and modify an action plan, and to maintain and apply two criteria simultaneously.

- The Stroop test is a common neuropsychological instrument to measure frontal lobe function by evaluating the inhibition of a pre-potent automatic response.

- The Stroop test consists of three different conditions that are based on the same number of stimuli.

- Simple and constrained phonemic fluency can assess high cognitive function including the executive domain specific to the frontal brain function. This task involves many complex operations such as finding the right words, initiating verbal responses, inhibiting responses that do not meet the criteria, correcting wrong outputs, and shifting attention to a new search.

\section{Ethical considerations}

All subjects signed an informed consent form. The study was approved by the ethical committee of "Grigore T. Popa” University of Medicine and Pharmacy from Iași, Romania. The study was conducted according to the Declaration of Helsinki. 


\section{Statistical methods}

Statistical analysis used SPSS version 19. Sample distribution was evaluated with the Kolmogorov-Smirnov test. We used parametric tests (Student's $t$-test) for continuous variables, independent, unequal variance assumed, and Pearson's method for correlations to compare the possible relationship between depression and QoL. Statistical significance level was set at a $95 \% \mathrm{CI}(P<0.05)$.

\section{Results}

\section{Subjects characteristics}

The mean age of acromegaly patients was $53.68 \pm 11.84$ and $55 \pm 12.28$ years for the control group $(P=0.739)$. The characteristics of acromegaly subjects along with the functional state of the pituitary are shown in Table 1 . Table 2 illustrates the demographic data of acromegaly and control group.

We used the Hamilton Depression Scale to assess the level of depression because depressed people may achieve lower scores on some cognitive measures due to psychomotor slowness. We further analyzed the correlation between the Hamilton Depression Scale scores and the AcroQoL global score because some questions are similar in both questionnaires. There was a significant negative relationship between the depression score obtained with Hamilton scale $(r=-0.45$; $P=0.005)$ and the global AcroQoL score. Subjects with a higher depression have a lower QoL as seen by a lower score on the AcroQoL.

\section{AcroQoL}

The average scores in the acromegaly group are significantly lower $(\mathrm{M}=60.80, \mathrm{SD}=13.37)$ than the control group $(\mathrm{M}=75.97, \mathrm{SD}=13.68, P=0.001)$.
We investigated the three dimensions in the AcroQoL. There are significant differences between the acromegaly group and the control group in terms of the physical effects $(P=0.001)$ and appearance $(P<0.001)$ but not for personal relationships $(P=0.421)$.

The average score for physical dimension in the acromegaly group was 20.89 ( $\mathrm{SD}=6.72)$ vs $28.52(\mathrm{SD}=6.2)$ for the controls; for appearance it was $20.47(\mathrm{SD}=5.91)$ vs 27.94 $(\mathrm{SD}=5.03)$ and for personal relationships $25.63(\mathrm{SD}=4.36)$ vs $27.10(\mathrm{SD}=6.58)$ for the acromegaly group and the controls, respectively.

\section{TMT}

The average time to solve the TMT part A was 58.94 seconds $(\mathrm{SD}=39.74)$ in the acromegaly group; the control group needed 45.5 seconds $(\mathrm{SD}=17.24)$ to solve the same part of the test $(P=0.195)$. The average time for solving TMT part B for subjects in the acromegaly group was $161.42(\mathrm{SD}=110.78)$ vs 74.77 ( $\mathrm{SD}=35.26)$ for the controls $(P=0.003)$.

We evaluated another parameter to analyze the differences between the two groups (acromegaly and control groups): the time difference needed to finish the two samples (time for part B minus time for part A). The average time B $-\mathrm{A}$ in the acromegaly group was 104 seconds $(\mathrm{SD}=87.28)$, which was significantly elevated $(P=0.002)$ vs the control group $(\mathrm{M}=29.27$ seconds; $\mathrm{SD}=25.93)$, suggesting that part $\mathrm{B}$ is more difficult to solve vs part A.

\section{Stroop test}

The Stroop test evaluated the time needed to read an incongruent list via ID (score difference) and IR (ratio score). The $t$-test for independent samples showed no significant

Table I Characteristics of the patients with controlled and uncontrolled acromegaly

\begin{tabular}{|c|c|c|}
\hline & Controlled $(n=8), M \pm S D$ & Uncontrolled $(n=I I), M \pm S D$ \\
\hline Age (years) & $56.875 \pm 8.084$ & $50.417 \pm 12.345$ \\
\hline Gender (F/M) & $6 / 2$ & $8 / 3$ \\
\hline Disease duration & $12.375 \pm 13.738$ & $8.583 \pm 8.489$ \\
\hline $\mathrm{GH}(\mathrm{ng} / \mathrm{dL})$ & $0.585 \pm 0.2853$ & $3.023 \pm 3.207$ \\
\hline IGF-I (ng/dL) & $\mid 99.875 \pm 91.279$ & $305.750 \pm 199.379$ \\
\hline Fasting blood glucose (mg/dL) & $113.264 \pm 25.602$ & $103.667 \pm 21.833$ \\
\hline $\mathrm{TSH}(\mu \mathrm{IU} / \mathrm{mL})$ & $0.832 \pm 0.777$ & $0.530 \pm 0.334$ \\
\hline fT4 (pg/mL) & $1.036 \pm 0.188$ & $1.029 \pm 0.124$ \\
\hline ACTH (pg/mL) & $29.313 \pm 11.912$ & $29.346 \pm 13.835$ \\
\hline Cortisol $(\mu \mathrm{g} / \mathrm{dL})$ & $8.683 \pm 4.389$ & $22.116 \pm 41.120$ \\
\hline Prolactin $(\mathrm{ng} / \mathrm{mL})$ & $3.780 \pm 2.161$ & $4.455 \pm 5.762$ \\
\hline Microadenoma, n (\%) & $3(37.5)$ & $0(0)$ \\
\hline Macroadenoma, n (\%) & $5(62.5)$ & $\mathrm{II}(100)$ \\
\hline
\end{tabular}

Abbreviations: ACTH, adrenocorticotropic hormone; F, female; FT4, free thyroxine; GH, growth hormone; IGF-I, insulin-like growth factor-I; M, male; TSH, thyroidstimulating hormone. 
differences between the acromegaly group and the control group. The average score difference was 79.44 ( $\mathrm{SD}=34.6)$ for the acromegaly group, and for the control group it was 71.42 ( $\mathrm{SD}=24.51), P=0.419$. The acromegaly group had a mean ratio score of $0.308(\mathrm{SD}=0.086)$, and the control group had $0.310(\mathrm{SD}=0.063)$. The differences are not significant $(P=0.949)$.

\section{Simple and constrained phonemic fluency}

The acromegaly group provided fewer words than the control group for letters "A" $(P=0.01)$ and "I" $(P=0.002)$ and no significant difference for letter " $\mathrm{P}$ " $(P=0.116)$. No significant differences were found for the constrained phonemic fluency. The acromegaly group achieved a mean of $4.73(\mathrm{SD}=3.12)$ and the control group 5.88 ( $\mathrm{SD}=2.84), P=0.250$.

All the neuropsychological test results are shown in Table 3 .

\section{Discussion}

The QoL of acromegaly patients is an important topic. Patient's expectations, living standards, and social integration are important concepts in evaluating the QoL. This study

Table 2 Demographic data of acromegaly and control groups

\begin{tabular}{|l|l|l|l|}
\hline & Patients & Controls & P-value \\
\hline Total N & 19 & 19 & \\
Female (\%) & 73.68 & 73.68 & \\
Male (\%) & 26.32 & 26.32 & \\
Age (years) & $53.68 \pm I I .84$ & $55 \pm 12.28$ & 0.739 \\
Educational level (years) & $11.315 \pm 2.38$ & $12.73 \pm 3.052$ & 0.118 \\
Hamilton scale score & $11.842 \pm 5.32$ & $10.421 \pm 4.63$ & 0.068 \\
\hline
\end{tabular}

showed that acromegaly is associated with a decreased QoL. The acromegaly group had global AcroQoL scores lower than the control group, suggesting a lower QoL. This study reports a global score of $60.8(\mathrm{SD}=13.37)$, which is similar to studies in Germany (62.1 [SD=18.2]), Belgium (67.1 [51.1-78.4]), and in France (59.8 [SD=17.9]). ${ }^{19-21}$

Although acromegaly has a negative impact on the body side and appearance, patients feel that their social life is not affected. This is important because social relations play a crucial role in psychological well-being. Subjects with acromegaly showed more visible physical effect including hypertension, polyarthralgia, osteoporosis, and kyphosis. Similar results were reported by Webb et al, but not Szcześniak et al, who reported worse social relationships than the general population. ${ }^{18,22,23}$

This study illustrates a significant relationship between dysexecutive syndrome and QoL. Unlike other acromegaly comorbid conditions such as cardiovascular disease, diabetes mellitus, osteoporosis, and sleep apnea, dysexecutive syndrome is an "invisible" and silent factor with a negative impact on QoL. Cognitive dysfunctions - particularly impairment of executive function - are subtle changes. Minimizing dysexecutive syndrome is an important step in improving QoL: cognitive-behavioral therapy including the "Think Healthy" technique had encouraging results. ${ }^{24}$

Executive function is one of the highest neurocognitive functions. This neuropsychological construct involves complex cognitive skills such as an ability to plan and organize with efficient working memory and flexibility. It is the capacity to reject inappropriate stimuli and to integrate past experiences into present actions.

Table 3 Neuropsychological test results for acromegaly group and healthy controls

\begin{tabular}{|c|c|c|c|c|}
\hline & \multirow[t]{2}{*}{ Items } & \multicolumn{3}{|l|}{ All patients } \\
\hline & & $\begin{array}{l}\text { Acromegaly } \\
\text { group (SD) }\end{array}$ & $\begin{array}{l}\text { Healthy } \\
\text { controls (SD) }\end{array}$ & $P$-value \\
\hline \multirow[t]{4}{*}{ AcroQoL } & Physical side & $20.89(6.72)$ & $28.52(6.2)$ & 0.001 \\
\hline & Appearance & $20.47(5.9 I)$ & $27.94(5.03)$ & 0.000 \\
\hline & Interpersonal relations & $25.63(4.36)$ & $27.1(6.58)$ & 0.634 \\
\hline & Total score & $60.8(13.37)$ & 75.97 (13.68) & 0.001 \\
\hline \multirow[t]{3}{*}{ TMT } & Part A (s) & $58.94(39.74)$ & 45.5 (I7.24) & 0.195 \\
\hline & Part B (s) & $161.42(110.78)$ & 74.77 (35.26) & 0.003 \\
\hline & B-A (s) & 104 & 29.27 & 0.002 \\
\hline Stroop test & ID & $79.44(34.6)$ & $71.42(24.5 \mathrm{I})$ & 0.419 \\
\hline \multirow[t]{3}{*}{ Simple phonemic fluency } & A & $8.47(4.62)$ & I3.05 (5.50) & 0.01 \\
\hline & I & $6.21(3.66)$ & $11.10(5.44)$ & 0.002 \\
\hline & $\mathrm{P}$ & $12.84(5.06)$ & I5.36 (4.59) & 0.116 \\
\hline Constrained phonemic fluency & & $4.73(3.12)$ & $5.88(2.84)$ & 0.250 \\
\hline
\end{tabular}

Notes: ID (the score difference) calculates the amount of time needed for naming the color from the incongruent list and the time needed recognizing the color from the congruent list; simple phonemic fluency was quantified by counting the number of words that patients were able to give for each of the three letters that we have provided: P, A, I; constrained phonemic fluency was evaluated by the number of words the subjects could provide in I minute beginning with the letter " $m$ " and having only four letters. Abbreviations: AcroQoL, Acromegaly Quality of Life Questionnaire; TMT, trail making test. 
Executive function is a new and exciting concept that has been recently investigated outside neuropsychology. Impaired executive function has been discovered in brain tumor, stroke in frontal lobes, Parkinson's disease, fibromyalgia syndrome, and type 1 diabetes mellitus. ${ }^{25,26}$

This study showed that acromegaly patients have a decreased executive function. The study explored different aspects of the executive function such as working memory and semantic inhibition. The acromegaly patients had lower scores than the controls in all tests with a statistically significant difference in the TMT part B and simple phonemic fluency.

The cognitive impairment is subtler. This might be because of the delay in diagnosing acromegaly. It takes 5-10 years on average and best 3.8 years. ${ }^{1-3}$ The patient is usually not aware of these changes and blames "ugly aging" associated with a deficit of memory, attention, as well as goal setting and execution.

Moreover, physicians tend to focus only on GH normalization and the treatment of the life-threatening consequences of the disease. Metabolic abnormalities such as diabetes, abnormal lipid profile, hypertension, heart failure or sleep apnea are severe complications that motivate the patient to seek adequate treatment, but cognitive dysfunctions can also decrease QoL.

Neurocognitive impairment in acromegaly has been evaluated in multiple studies with contradictory results. Sievers et $\mathrm{al}^{27}$ reported that patients with acromegaly have a decrease in cognitive functioning as follows: $33 \%$ with reduced attention, $24.1 \%$ with reduced memory, and $16.7 \%$ with reduced executive function; $67.3 \%$ could not reach the cutoff level in at least one test. ${ }^{27}$ Leon-Carrion et al proved that short-term and long-term memory are most severely impaired, and the prefrontal and middle temporal cortices have decreased activity. ${ }^{28}$

Neuropsychological tests revealed that lower memory scores are associated with untreated disease duration with few deficits in executive functions. ${ }^{4}$ Tiemensma et $\mathrm{al}^{29}$ reported no cognitive dysfunction in patients with long-term cured acromegaly. ${ }^{29}$

Most studies that have analyzed cognitive function report that patients diagnosed with acromegaly achieve lower scores than control groups on memory and executive functioning. ${ }^{2,28,30-32}$ Tanriverdi et al ${ }^{32}$ used cognitive electroencephalographic (EEG)-evoked potentials to show that there is a reduction of $\mathrm{P} 300$ amplitudes in brain areas in acromegaly patients. These areas are important for cognitive functioning and include the inferior parietal lobe, frontal lobe, hippocampus, and medial temporal lobe. ${ }^{30}$ An MRI study compared 44 patients with acromegaly to healthy controls and found that acromegaly led to alterations of the macroscopic brain tissue architecture that appear during the first 10 years of the disease; patients had increased gray matter volume $(+3.7 \%)$ and white matter volume $(+5.1 \%)$, which might be due to excessive GH and IGF-1 on neuronal or glial cells. ${ }^{4}$ Low-resolution brain electromagnetic tomography (LORETA) was used to estimate the electrical brain activity in 16 untreated acromegaly patients vs 16 matched controls - there was decreased activity in the right inferior frontal lobe alpha, the right dorsolateral prefrontal cortex $\beta-2$, and the right parahippocampal cortex $\beta-3$ waves. ${ }^{28}$ A combination of EEG and LORETA in acromegaly patients showed lower $\alpha$ and $\beta$ cortical activity in the left temporal cortex vs healthy controls. ${ }^{2}$

\section{Conclusion}

This study suggests that executive functioning is affected in acromegaly. These results indicate that clinicians should consider cognitive tests to evaluate executive functioning in addition to standard screening for comorbidities. We highlight the benefit of an interdisciplinary psychological intervention to acromegaly disease that can improve the patients' QoL.

Further studies with a larger number of subjects and a larger battery of tests are needed to investigate the cognitive functions in acromegaly. Another aspect that might possibly be evaluated is a correlation between the degree of cognitive dysfunction with the disease-specific hormone levels (GH, IGF-1, etc) and imaging parameters (LORETA).

\section{Acknowledgments}

This research did not receive any specific grant from funding agencies in public, commercial, or non-for-profit sectors. We thank Professor Carmen Vulpoi for her support.

\section{Disclosure}

The authors report no conflicts of interest in this work.

\section{References}

1. Melmed S. Acromegaly pathogenesis and treatment. J Clin Invest. 2009;119(11):3189-3202.

2. Martín-Rodríguez JF, Madrazo-Atutxa A, Venegas-Moreno E, et al. Neurocognitive function in acromegaly after surgical resection of GH-secreting adenoma versus naïve acromegaly. PLoS One. 2013;8(4):e60041.

3. Niculescu DA, Baciu IF, Capatina C, et al. Acromegaly treatment in Romania. How close are we to disease control? Endokrynol Pol. 2017;68(5): 519-523.

4. Sievers C, Sämann PG, Dose T, et al. Macroscopic brain architecture changes and white matter pathology in acromegaly: a clinicoradiological study. Pituitary. 2009;12(3):177-185. 
5. Åberg ND, Brywe KG, Isgaard J. Aspects of growth hormone and insulin-like growth factor-I related to neuroprotection, regeneration, and functional plasticity in the adult brain. ScientificWorldJournal. 2006;6:53-80.

6. Hallberg M, Nyberg F. Growth hormone receptors in the brain and their potential as therapeutic targets in central nervous system disorders. Open Endocrinol J. 2012;6(1):27-33.

7. Lai Z, Roos P, Zhai O, et al. Age-related reduction of human growth hormone-binding sites in the human brain. Brain Res. 1993;621(2): 260-266.

8. Pereira AM. Neuropsychological functioning in acromegaly: towards identification of modifiable factors to improve long-term care after remission. Endocrine. 2015;50(3):523-525.

9. Suchy Y. Executive functioning: overview, assessment, and research issues for non-neuropsychologists. Ann Behav Med. 2009;37(2):106-116.

10. Salthouse TA. What cognitive abilities are involved in trail-making performance? Intelligence. 2011;39(4):222-232.

11. Bowie CR, Harvey PD. Administration and interpretation of the trail making test. Nat Protoc. 2006;1(5):2277-2281.

12. Heflin LH, Laluz V, Jang J, Ketelle R, Miller BL, Kramer JH. Let's inhibit our excitement: the relationships between Stroop, behavioral disinhibition, and the frontal lobes. Neuropsychology. 2011;25(5):655-665.

13. Nyberg F. Growth hormone in the brain: characteristics of specific brain targets for the hormone and their functional significance. Front Neuroendocrinol. 2000;21(4):330-348.

14. Deijen JB, Arwert LI, Drent ML. The GH/IGF-I Axis and cognitive changes across a 4-year period in healthy adults. ISRN Endocrinol. 2011;2011:1-6.

15. Al-Delaimy WK, von Muhlen D, Barrett-Connor E. Insulinlike growth factor-1, insulinlike growth factor binding protein-1, and cognitive function in older men and women. JAm Geriatr Soc. 2009;57(8):1441-1446.

16. Burman P, Hetta J, Wide L, Månsson JE, Ekman R, Karlsson FA. Growth hormone treatment affects brain neurotransmitters and thyroxine [see comment]. Clin Endocrinol. 1996;44(3):319-324.

17. Crespo I, Webb SM. Perception of health and cognitive dysfunction in acromegaly patients. Endocrine. 2014;46(3):365-367.

18. Szcześniak DM, Jawiarczyk-Przybyłowska A, Matusiak Ł, et al. Is there any difference in acromegaly and other chronic disease in quality of life and psychiatric morbidity? Endokrynol Pol. 2017;68(5):524-532.

19. Psaras T, Honegger J, Gallwitz B, Milian M. Are there gender-specific differences concerning quality of life in treated acromegalic patients? Exp Clin Endocrinol Diabetes. 2011;119(5):300-305.

20. T'sjoen G, Bex M, Maiter D, Velkeniers B, Abs R. Health-related quality of life in acromegalic subjects: data from AcroBel, the Belgian registry on acromegaly. Eur J Endocrinol. 2007;157(4):411-417.
21. Matta MP, Couture E, Cazals L, Vezzosi D, Bennet A, Caron P. Impaired quality of life of patients with acromegaly: control of GH/IGF-I excess improves psychological subscale appearance. Eur J Endocrinol. 2008;158(3):305-310.

22. Webb SM, Badia X, Surinach NL, Spanish AcroQol Study Group. Validity and clinical applicability of the acromegaly quality of life questionnaire, AcroQoL: a 6-month prospective study. Eur J Endocrinol. 2006;155(2):269-277.

23. Webb SM, Badia X. Quality of life in growth hormone deficiency and acromegaly. Endocrinol Metab Clin North Am. 2007;36(1):221-232.

24. Kunzler LS, Naves LA, Casulari LA. Cognitive-behavioral therapy improves the quality of life of patients with acromegaly. Pituitary. 2018;21(3):323-333.

25. Muñoz Ladrón de Guevara C, Fernández-Serrano MJ, Reyes del Paso GA, Duschek S. Executive function impairments in fibromyalgia syndrome: relevance of clinical variables and body mass index. PLoS One. 2018;13(4):e0196329.

26. Perez KM, Patel NJ, Lord JH, et al. Executive function in adolescents with Type 1 diabetes: relationship to adherence, glycemic control, and psychosocial outcomes. J Pediatr Psychol. 2017;42(6):636-646.

27. Sievers C, Sämann PG, Pfister H, et al. Cognitive function in acromegaly: description and brain volumetric correlates. Pituitary. 2012;15(3): 350-357.

28. Leon-Carrion J, Martin-Rodriguez JF, Madrazo-Atutxa A, et al. Evidence of cognitive and neurophysiological impairment in patients with untreated naive acromegaly. J Clin Endocrinol Metab. 2010;95(9): 4367-4379.

29. Tiemensma J, Biermasz NR, van der Mast RC, et al. Increased psychopathology and maladaptive personality traits, but normal cognitive functioning, in patients after long-term cure of acromegaly. $J$ Clin Endocrinol Metab. 2010;95(12):365-367.

30. Brummelman P, Koerts J, Dullaart RP, et al. Effects of previous growth hormone excess and current medical treatment for acromegaly on cognition. Eur J Clin Invest. 2012;42(12):1317-1324.

31. Yedinak CG, Fleseriu M. Self-perception of cognitive function among patients with active acromegaly, controlled acromegaly, and nonfunctional pituitary adenoma: a pilot study. Endocrine. 2014;46(3): 585-593.

32. Tanriverdi F, Yapislar H, Karaca Z, Unluhizarci K, Suer C, Kelestimur F. Evaluation of cognitive performance by using P300 auditory event related potentials (ERPs) in patients with growth hormone $(\mathrm{GH})$ deficiency and acromegaly. Growth Horm IGF Res. 2009;19(1):24-30.
Psychology Research and Behavior Management

\section{Publish your work in this journal}

Psychology Research and Behavior Management is an international, peerreviewed, open access journal focusing on the science of psychology and its application in behavior management to develop improved outcomes in the clinical, educational, sports and business arenas. Specific topics covered in the journal include: Neuroscience, memory and decision making; Behavior

\section{Dovepress}

modification and management; Clinical applications; Business and sports performance management; Social and developmental studies; Animal studies. The manuscript management system is completely online and includes a very quick and fair peer-review system, which is all easy to use. Visit http://www. dovepress.com/testimonials.php to read real quotes from published authors. 